Proceedings of the

International Geometry Center

Vol. 13, no. 1 (2020) pp. 23-34

\title{
Про існування деформацій овалоїдів
}

\author{
Т. Ю. Подоусова, Н. В. Вашпанова
}

\begin{abstract}
In this paper, general infinitesimal deformations of higher orders of simply connected surfaces are considered in three-dimensional Euclidean space $E_{3}$, which are important in the study of their continuous deformations. The problem of finding the displacement vectors of these strains is reduced to the study and solution of a system of $n$ equations (or basic equations) of general infinitesimal deformations of finite order $n$, which are obtained with respect to an arbitrarily chosen coordinate system on the surface. It is shown that for closed surfaces of positive Gaussian curvature, the mathematical model of this problem in the conjugate-isothermal coordinate system is a system of $n$ heterogeneous equations of a complex form, which in the case of an ovaloid is reduced to an system of $n$ integral equations. Using tensor methods, the apparatus of the theory of generalized analytic functions, and methods of functional analysis, it is proved that a regular ovaloid in $E_{3}$ "as a whole" admits a general infinitesimal deformation of finite order $n$, which is uniquely determined by predefined $3 n$ functions. Their geometric meaning is found: setting them is equivalent to setting the values of the variations of the normal unit vector and the area element up to the order $n$ inclusively. The strain vector fields are determined up to constant vectors. It was established that the ovaloid will be tough with respect to the general infinitesimal deformations of finite order $n$ if and only if all values of the variations of the normal unit vector and the area element up to order $n$ inclusive are identically equal to zero. A sphere of radius $R$ is considered as a confirming example of the surface. The displacement vectors are found in explicit form.
\end{abstract}

Анотація. У даній роботі у тривимірному евклідовому просторі $E_{3}$ розглядаються загальні нескінченно малі (н.м.) деформації вищих порядків однозв'язних поверхонь, які мають важливе значення при вивченні їх неперервних деформацій. Завдання знаходження векторів зсуву цих деформацій зводиться до дослідження і розв'язку системи $n$ рівнянь (або основних рівнянь) загальних н.м. деформацій скінченого порядку $n$, які

Ключові слова: infinitesimal deformation, regular ovaloid 
отримані відносно довільно обраної на поверхні системи координат. Показано, що для замкнутих поверхонь додатньої гаусової кривини математичною моделлю цього завдання в сполучено-ізотермічній системі координат буде система $n$ неоднорідних рівнянь комплексного виду, яка у випадку овалоїда приводиться до системи $n$ інтегральних рівнянь.

Використовуючи тензорні методи, апарат теорії узагальнених аналітичних функцій і методи функціонального аналізу, доведено, що регулярний овалоїд в $E_{3}$ "в цілому" допускає загальну н.м. деформацію скінченого порядку $n$, яка однозначно визначається заздалегідь заданими $3 n$ функціями. Знайдений їх геометричний зміст: завдання їх рівносильно завданням значень варіацій орта нормалі і елемента площі до порядку $n$ включно. Векторні поля деформації при цьому визначаються з точністю до постійних векторів.

Встановлено, що овалоїд буде жорстким щодо загальних н.м. деформацій скінченого порядку $n$ тоді і тільки тоді, коли всі значення варіацій орта нормалі і елемента площі до порядку $n$ включно тотожно рівні нулю. В якості прикладу поверхні, яка підтверджує отриманий результат, розглянута сфера радіуса $R$. Вектори зміщень при цьому знайдені в явному вигляді.

Дослідження загальних нескінченно малих (з.н.м) деформацій вищих порядків поверхонь є важливим етапом у вивченні їх неперервних деформацій. Н.м. деформації вищих порядків поверхонь при певних обмеженнях вивчалися багатьма авторами $[11,13,16,10,14,17]$. Технічні складнощі, які виникають при високих порядках деформації, та потреби практичного застосування, приводили до необхідності спеціалізації як деформації, так і поверхонь, що деформуються. Зокрема, вивчалися н.м. деформації першого порядку зі збереженням елемента площі [6, 7], геодезичних ліній [2], спеціальних векторних полів [1] та інших геометричних об'єктів $[3,4]$.

Предметом дослідження в даній роботі є регулярний овалоїд у тривимірному евклідовому просторі $E_{3}$. Підвищенний інтерес до цієї поверхні зумовлений тим, що вона має широке застосування, насамперед, у газовій динаміці при вивченні поверхонь плівок та оболонок. Саме міцність або жорсткість будь-якої опуклої замкненої поверхні характеризується наявністю або відсутністю н.м. деформації, які описують цю поверхню. Слід відзначити, що деякі н.м. деформації овалоїдів вивчалися, наприклад, в [9, 5, 18, 12]. В роботах [15] і [8] автори різними шляхами прийшли до наступного висновку: всякий регулярний овалоїд допускає н.м. деформацію першого порядку зі збереженням елемента площі поверхні і однозначно визначається заздалегідь заданими двома функціями, кожна від двох змінних, що є частковим випадком результату, отриманого у даній роботі (Теорема 1). 


\section{1. ОСНовні РІвНЯНня 3.н.м. ДЕФОРМАЦІй ВИщИХ ПОРЯДКІВ} ПОВЕРХОНЬ

Нехай у $E_{3}$-просторі задана однозв' язна регулярна поверхня $S$ класу $C^{3}$ з радіус-вектором $\mathbf{r}=\mathbf{r}\left(x^{1}, x^{2}\right)$ і гомеоморфна області $G$ площини (або всій площині).

Розглянемо н.м. деформацію скінченного $n$-ого порядку поверхні $S$ вигляду [11]:

$$
\mathbf{r}^{*}\left(x^{1}, x^{2}, t\right)=\mathbf{r}\left(x^{1}, x^{2}\right)+\sum_{k=1}^{n} t^{k} \mathbf{y}^{k}\left(x^{1}, x^{2}\right)
$$

3 регулярними полями зміщення $\stackrel{k}{\mathbf{y}}\left(x^{1}, x^{2}\right)$ класу $C^{2}$, частинні похідні яких розкладемо за базисом $\mathbf{r}_{i}=\frac{\partial \mathbf{r}}{\partial x^{i}}, \mathbf{n}:$

$$
\mathbf{y}_{i}^{k}=c_{i \alpha} \stackrel{k}{\hat{T}^{\alpha \beta}} \mathbf{r}_{\beta}+c_{i \alpha} \stackrel{k}{T}^{\alpha} \mathbf{n}, \quad k=\overline{1, n},
$$

де $c_{11}=c_{22}=0, c_{12}=-c_{21}=\sqrt{g}, g=g_{11} g_{22}-g_{12}^{2}, g_{i j}-$ метричний тензор, $\mathbf{n}$ - орт нормалі $S$, а $\hat{T}^{\alpha \beta}, \stackrel{k}{T^{\alpha}}$ - деякі тензорні поля на $S$. Індекси всюди набувають значень 1, 2.

Представимо $\hat{T}^{\alpha \beta}$ у вигляді суми симетричного $T^{\alpha \beta}$ i кососиметричного $\stackrel{k}{\mu} c^{\alpha \beta}$ тензорів:

$$
\stackrel{k}{\hat{T}^{\alpha \beta}}=\stackrel{k}{k} T^{\alpha \beta}+\stackrel{k}{\mu} c^{\alpha \beta}, \quad k=\overline{1, n},
$$

де $\stackrel{k}{\mu}\left(x^{1}, x^{2}\right)$ - деякі функції класу $C^{2}, c^{\alpha \beta}=g^{\alpha i} g^{\beta j} c_{i j}, g^{\alpha \beta}$ - елементи матриці оберненої до матриці $\left\|g_{i j}\right\|$.

Розглянувши умови інтегровності (1.1) і скориставшись дериваційними формулами теорії поверхонь [11], отримаємо наступну систему рівнянь:

$$
\stackrel{k}{T_{, \alpha}^{\alpha i}}=b_{\alpha}^{i} \stackrel{k}{T^{\alpha}}+\stackrel{k}{\mu} c^{i \alpha}, \quad \stackrel{k}{T^{\alpha \beta}} b_{\alpha \beta}=-\stackrel{k}{T}_{, \alpha}^{\alpha}, \quad k=\overline{1, n} .
$$

Тут $b_{\alpha}^{i}=g^{i \beta} b_{\alpha \beta}, b_{\alpha \beta}-$ коефіцієнти другої квадратичної форми $S$, а комою позначено коваріантне диференціювання на базі $g_{i j}$.

Через будь-який розв'язок системи рівнянь (1.3) із (1.1) знайдемо вектори зміщення $\stackrel{k}{\mathbf{y}}\left(x^{1}, x^{2}\right)$, які будуть однозначними функціями в силу однозв'язності поверхні $S$ :

$$
\stackrel{k}{\mathbf{y}}=\int_{M_{0} M}\left(c_{i \alpha}\left(\stackrel{k}{T^{\alpha \beta}}+\stackrel{k}{\mu} c^{\alpha \beta}\right) \mathbf{r}_{\beta}+c_{i \alpha} T^{\alpha} \mathbf{n}\right) d x^{i}+\stackrel{k}{\mathbf{y}_{0}}, \quad k=\overline{1, n},
$$


де інтеграли обчислюються вздовж довільної спрямної кривої, що лежить на $S$ і з'єднує фіксовану точку $M_{0}$ із змінною точкою $M$, а $\mathbf{y}_{0}-$ постійні вектори.

Якщо векторні поля $\stackrel{k}{\mathbf{y}}=$ const, то будемо говорити, що поверхня $\mathrm{\epsilon}$ жсрсткою відносно з.н.м. деформацій, а сама деформація є тривіальною.

Таким чином, задача знаходження векторів зміщення з.н.м. деформації еквівалентна розв'язуванню системи рівнянь (1.3). Тому (1.3) називатимемо основними рівняннями з.н.м. деформацій вищих порядків поверхонь.

2. КОМПЛЕКСНИЙ ВИГЛЯД СИСТЕМИ РІВНЯНЬ (1.3) ДЛЯ ОВАЛЬНИХ ПОВЕРХОНЬ

Розглянемо всюди замкнену поверхню $S$ додатньої гаусової кривини $\left(K \geqslant K_{0}>0\right.$ (в $\left.G\right), K_{0}=$ const) класу $D_{l+5, p}, l \geqslant 0, p>2$. (Позначення класів функцій запозичені у [9]).

Введемо до розгляду сукупність комплексних функцій $\stackrel{k}{\omega}$ комплексної змінної $z=x^{1}+i x^{2}, i^{2}=-1$ :

$$
\stackrel{k}{\omega}=g \sqrt[4]{K}\left(\stackrel{k}{\tilde{T}^{11}}-i \stackrel{k}{\tilde{T}^{12}}\right)
$$

де

$$
\stackrel{k}{T}^{i j}=\stackrel{k}{T^{i j}}+\frac{{ }^{T}}{2 H}{ }_{, \alpha}^{i j}
$$

і $H(H \neq 0)$ - середня кривина $S$. Віднесемо поверхню $S$ до сполученоізотермічної системи координат, відносно якої систему рівнянь (1.3) за методом Векуа I.Н. приведемо до вигляду, [9, p.5, §6, с.342]:

$$
\frac{\partial \stackrel{k}{\omega}}{\partial \bar{z}}-\bar{B} \frac{k}{\omega}=\stackrel{k}{F}\left(\stackrel{k}{T^{1}}, \stackrel{k}{T^{2}}, \stackrel{k}{\mu}\right), k=\overline{1, n}
$$

Тут

$$
\frac{\partial \stackrel{k}{\omega}}{\partial \bar{z}}=\frac{1}{2}\left(\frac{\partial \omega}{\partial x^{1}}+i \frac{\partial \stackrel{k}{\omega}}{\partial x^{2}}\right), \quad \stackrel{k}{\widetilde{T}^{11}}=-\stackrel{k}{\widetilde{T}^{22}}, \quad \stackrel{k}{\widetilde{T}^{12}}=\stackrel{k}{\widetilde{T}^{21}}
$$


а функції $\stackrel{k}{F}$ мають наступне представлення:

$$
\begin{aligned}
\stackrel{k}{F}=\frac{\sqrt[4]{K}}{2} & {\left[\left(g_{22}+i g_{12}\right)\left(\left(\frac{T_{, \alpha}^{\alpha}}{2 H}\right)_{1}+b_{11} T^{1}\right)\right]-} \\
& -\frac{\sqrt[4]{K}}{2}\left[\left(g_{12}+i g_{11}\right)\left(\left(\frac{T_{, \alpha}^{\alpha}}{2 H}\right)_{2}+b_{11} T^{2}\right)-\left(k_{\mu_{2}}+i \mu_{1}\right)\right]
\end{aligned}
$$

Слід відзначити, що функція $B$, яка входить в кожне рівняння системи (2.3) має такий же вигляд, як і у рівнянні Векуа I. Н., яке отримано при дослідженні н.м. згинань першого порядку поверхонь додатної гаусової кривини, [9, р.5, §3, с. 323].

Крім відомих функцій точки $S$ і шуканих комплексних функцій $\stackrel{k}{\omega}$ праві частини рівнянь (2.3) містять і функції $\stackrel{k}{T}, \stackrel{k}{\mu},(k=\overline{1, n})$. Для забезпечення визначенності системи рівнянь (2.3) будемо рахувати їх заданими функціями в замкненій області $G$.

\section{3. ПРО ІСНУВАННЯ З.Н.М. ДЕФОРМАЦІЙ ВИЩИХ ПОРЯДКІВ ОВАЛОЇДІВ}

Задача про існування з.н.м. деформацій вищих порядків овальних поверхонь зводиться до дослідження і розв'язування системи рівнянь $(2.3)$.

При вивченні з.н.м. деформацій скінченого порядку $n$ овалоїдів суттєвим є дослідження поведінки функцій $B, \stackrel{k}{F}$ в точці $z=\infty$ розширеної комплексної площини.

Відобразимо $S$ на площину $E$. Тоді асимптотична поведінка поблизу нескінченно віддаленої точки $z=\infty$ для наступних функцій матиме вигляд, $[9$, р.6, $\S 5$, с. 471$]$ :

$$
g_{\alpha \beta}, \quad g=O\left(|z|^{-4}\right), \quad \mathbf{r}_{z}, \quad \mathbf{r}_{\bar{z}}, \quad B(z), \quad P_{z}, \quad P_{\bar{z}}=O\left(|z|^{-2}\right),
$$

де $P$ - деяке неперервно-диференційовне скалярне чи векторне поле на $S$.

Справедлива наступна

Теорема 3.1. Замкнена всюди додатної гаусової кривини поверхня $S$ класу $D_{l+5, p}, l \geqslant 0, p>2$ «у иілому» допускае з.н.м. деформачію скінченого $n$-го порядку з векторами зміщення $\mathbf{y}^{k}\left(x^{1}, x^{2}\right)$ класу $C_{\lambda}^{l+3}(E)$, $\lambda=\frac{p-2}{p}$, яка однозначно визначається заздалегідь заданими функиія-

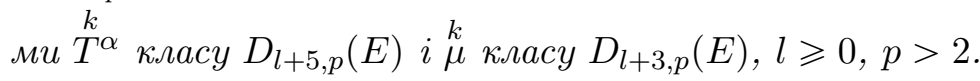


Доведення. Оскільки за умовою теореми $S \in D_{l+5, p}(E)$, то функції $([9$, p. $2, \S 6$, c. 93$])$ :

$$
\sqrt{g}, b_{\alpha \beta}, K, H \in D_{l+3, p}(E), \quad B \in D_{l+2, p}(E), \quad l \geqslant 0, p>2 .
$$

Задамо на овалоїді функції $\stackrel{k}{T^{\alpha}}$ i $\stackrel{k}{\mu}$ таким чином, щоб

$$
\begin{aligned}
& \stackrel{k}{T^{\alpha}} \in D_{l+5, p}(E), l \geqslant 0, p>2, k=\overline{1, n}, \\
& \stackrel{k}{\mu} \in D_{l+3, p}(E), l \geqslant 0, p>2, k=\overline{1, n},
\end{aligned}
$$

і поблизу нескінченно віддаленої точки $z=\infty$ мали наступні оцінки:

$$
\stackrel{k}{T^{\alpha}}=O\left(|z|^{2}\right), \quad \stackrel{k}{\mu}=O(1), k=\overline{1, n} .
$$

Тоді згідно з означенням коваріантної похідної функцій $\stackrel{k}{T_{, \alpha}^{\alpha}}$, дістанемо

$$
\stackrel{k}{T_{, \alpha}^{\alpha}} \in D_{l+3, p}(E), l \geqslant 0, p>2, k=\overline{1, n} .
$$

Скориставшись диференціальними властивостями відомих і заданих функцій із (2.5) випливає, що

$$
\stackrel{k}{F} \in D_{l+2, p}(E), \quad l \geqslant 0, p>2 .
$$

Прийнявши до уваги оцінки поблизу точки $z=\infty$ відомих і заданих функцій із (2.5) дістанемо

$$
\stackrel{k}{F}=O\left(|z|^{-6}\right), \quad k=\overline{1, n} .
$$

Помноживши рівності (1.1) на $c^{i s} \mathbf{r}^{t}$, знайдемо:

$$
\hat{T}^{s t}=c^{i s} \mathbf{r}^{t^{k}} \mathbf{y}_{i}, \quad k=\overline{1, n} .
$$

Тоді в силу оцінок відомих і заданих функцій поблизу $z=\infty$ із (1.2), матимемо

$$
\stackrel{k}{T^{s t}}=O\left(|z|^{4}\right) .
$$

Отже, із рівностей $(2.1)$ згідно $(2.2)$, знайдемо поведінку функцій $\stackrel{k}{\omega}$ в околі нескінченно віддаленої точки $z=\infty$ :

$$
\stackrel{k}{\omega}=O\left(|z|^{-4}\right) .
$$

Покажемо тепер, що система рівнянь (2.3) має розв'язок $\stackrel{1}{\omega}, \stackrel{2}{\omega}, \ldots, \stackrel{n}{\omega}$, який на нескінченності задовольняе умовам (3.5).

Відомо, що $B \in L_{p, 2}, p>2([9, \mathrm{p} .2, \S 6$, c.103]). Тоді в силу оцінок (3.1), $(3.4),(3.5)$ та згідно теореми 1.23 із [9], кожне рівняння системи (2.3) 
при конкретному значенні $k$ зводиться до інтегрального рівняння ([9, p.3, §2, с.116]), тобто (2.3) набуде вигляду:

$$
\stackrel{k}{\omega}-P_{E} \stackrel{k}{\omega}=T_{E} \stackrel{k}{F}\left(\stackrel{k}{T^{1}}, \stackrel{k}{T^{2}}, \stackrel{k}{\mu}\right), k=\overline{1, n},
$$

де

$$
\begin{gathered}
T_{E} \stackrel{k}{F}=-\frac{1}{\pi} \iint_{E} \frac{\stackrel{k}{F}(\xi) d \zeta d \eta}{\xi-z}, \quad \xi=\zeta+i \eta \\
P_{E} \stackrel{k}{\omega}=-\frac{1}{\pi} \iint_{E} \frac{\bar{B} \frac{k}{\omega} d \zeta d \eta}{\xi-z} .
\end{gathered}
$$

При кожному конкретному значенні $k$ оператор $P_{E} \stackrel{k}{\omega}$ є цілком неперервним в $L_{q, 0}(E), q \geqslant \frac{p}{p-1}([9$, теорема 1.26$])$. Згідно теореми 3.11 із [9] відповідні однорідні рівняння

$$
\stackrel{k}{\omega}-P_{E} \stackrel{k}{\omega}=0, \quad k=\overline{1, n}
$$

для неоднорідного рівняння із (3.6) при конкретному значенні $k$ мають тільки тривіальні розв'язки $\stackrel{k}{\omega}=0$ всюди на $E$.

Із [9, теорема 1.23] випливає, що права частина кожного неоднорідного рівняння при конкретному значенні $k$ із (3.6) належить класу $L_{q, 0}(E), q \geqslant \frac{p}{p-1}$.

Тоді кожне неоднорідне рівняння із (3.6) при конкретному значенні $k$ має розв'язок, притому єдиний в класі $L_{q, 0}(E), q \geqslant \frac{p}{p-1}([9]$, p.3, §5, c.130).

3 іншого боку, в силу (3.2), (3.3), матимемо

$$
\stackrel{k}{\omega} \in D_{l+3, p}(E), l \geqslant 0, p>2 .
$$

Отже, при заданих функціях $\stackrel{k}{T^{\alpha}}, \stackrel{k}{\mu}$ розв' язок системи рівнянь (3.6) існує всюди на площині $E$, єдиний і належить класу $D_{l+3, p}(E), l \geqslant 0, p>2$. В силу рівностей (2.1) та згідно з диференціальними властивостями

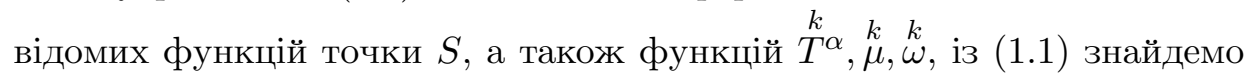
$\mathbf{y}_{i} \in D_{l+3, p}(E), l \geqslant 0, p>2$. Звідси випливає, що вектори зміщення $\stackrel{k}{\mathbf{y}} \in C_{\lambda}^{l+3}(E), \lambda=\frac{p-2}{p}([9, \mathrm{p} .2, \S 6, \mathrm{c.101}])$. Теорему доведено. 
Лема 3.2. [Про жорсткість овалоїдів] Овалоїд е жорстким відносно з.н.м. деформачій п-ого порлдку тоді і тільки тоді, коли функціӥ $\stackrel{k}{T^{\alpha}}, \stackrel{k}{\mu},(k=\overline{1, n})$ тотожно дорівнюють нулю.

Доведення. Достатність. Нехай $\stackrel{k}{T^{\alpha}}=\stackrel{k}{\mu}=0,(k=\overline{1, n})$. Тоді із (2.5) можемо знайти функції $\stackrel{k}{F}=0$. При цьому кожне рівняння системи $(2.3)$ буде однорідним, і у випадку овалоїда воно матиме єдиний нульовий розв'язок $\stackrel{k}{\omega}=0,(k=\overline{1, n}),[9$, p.5, $\S 7$, с. 356$]$.

Враховуючи $(2.1),(2.2),(1.2)$ із (1.1) знайдемо $\mathbf{y}_{i}^{k}=0,(k=\overline{1, n})$. Це означає, що $\mathbf{y}^{k}=$ const, а отже, овалоїд є жорстким відносно з.н.м. деформації $n$-порядку.

Необхідністъ. Нехай вектори зміщення $\stackrel{k}{\mathbf{y}}=$ const, $(k=\overline{1, n})$ при 3.н.м. деформації скінченного $n$-го порядку овалоїда є постійними. Тоді $\stackrel{k}{\mathbf{y}_{i}}=0,(k=\overline{1, n})$. Із рівностей (1.1) знайдемо, що $\stackrel{k}{\hat{T}^{i j}}=0$ i $\stackrel{k}{T^{\alpha}}=0$. Отже, функції $\stackrel{k}{F}$ із $(2.5)$ матимуть вигляд

$$
\stackrel{k}{F}=\frac{\sqrt[4]{K}}{2}\left(\begin{array}{l}
k \\
\mu_{2}
\end{array}+i \mu_{1}^{k}\right), \quad k=\overline{1, n} .
$$

Скориставшись (1.2), (2.2) із (2.1) знайдемо $\stackrel{k}{\omega}=i{\sqrt[4]{g^{2} K}}^{k}$. Підставивши знайдені вирази для $\stackrel{k}{\omega}$ і $\stackrel{k}{F}$ в кожне рівняння системи рівнянь (2.3), яке буде тотожністю тільки у випадку $\stackrel{k}{\mu}=0, k=\overline{1, n}$. Лему доведено.

\section{4. ГЕОМЕТРИЧНИЙ ЗМІСТ ФУНКЦІй $\stackrel{k}{T^{\alpha}}$ I $\stackrel{k}{\mu}$}

При з.н.м. деформації $S$ скінченного порядку $n k$-ті варіації $\delta^{k} d \sigma$ елемента площі $d \sigma$ поверхні мають вигляд [10]:

$$
\delta^{k} d \sigma=g\left(g^{i j} \varepsilon_{i j}^{k}+c^{i \alpha} c^{j \beta} \sum_{m=1}^{k-1}{\underset{\varepsilon}{\varepsilon_{i j}}}^{k-m}{ }_{\varepsilon}^{k \beta}\right) d \sigma,
$$

де варіації коефіцієнтів першої квадратичної форми $S$ мають наступне представлення:

$$
2 \varepsilon_{i j}^{k}=\delta^{k} g_{i j}=\stackrel{k}{\hat{T}^{\alpha \beta}}\left(c_{i \alpha} g_{j \beta}+c_{j \alpha} g_{i \beta}\right)+c_{i \alpha} c_{j \beta} A^{\alpha \beta},
$$

причому

$$
A^{k \beta}=\sum_{m=1}^{k-1}\left(\begin{array}{lr}
m k-m & m^{k-m} \\
\hat{T}^{\alpha s} \hat{T}^{\beta t} & g_{s t}+T^{\alpha} T^{\beta}
\end{array}\right) .
$$


В силу рівностей (1.2) попередній вираз для $\delta^{k} d \sigma$ набуде вигляду:

$$
\delta^{k} d \sigma=(-2 \stackrel{k}{\mu} g+\stackrel{k}{N}) d \sigma
$$

де

$$
\stackrel{k}{N}=g\left(\frac{1}{2} g_{\alpha \beta} A^{\alpha \beta}+c^{i \alpha} c^{j \beta} \sum_{m=1}^{k-1} \stackrel{m}{\varepsilon}_{i j}{ }^{k-m}{ }_{\alpha \beta}\right)
$$

Звідси знайдемо формулу для функцій $\stackrel{k}{\mu}$ :

$$
\stackrel{k}{\mu}=\frac{1}{2 g}\left(\stackrel{k}{N}-\frac{\delta^{k} d \sigma}{d \sigma}\right) .
$$

Отже, функції $\stackrel{k}{\mu}$ можна задати варіаціями елемента площі $S$.

Оскільки орт нормалі має представлення

$$
\mathbf{n}=\frac{1}{2} c^{i j} \mathbf{r}_{i} \times \mathbf{r}_{j},
$$

то $k$-ті варіації орта нормалі набудуть вигляду

$$
\delta^{k} \mathbf{n}=c^{i j}\left(\mathbf{r}_{i} \times \stackrel{k}{\mathbf{y}_{j}}+\mathbf{r}_{j} \times \stackrel{k}{\mathbf{y}_{i}}\right)+\frac{1}{2} \delta^{k} c^{i j} c_{i j} \mathbf{n}+\stackrel{k}{\mathbf{s}}, \quad k=\overline{1, n}
$$

де

$$
\stackrel{k}{\mathbf{s}}=\sum_{m=1}^{k-1}\left(\delta^{m} c^{i j}\left(\begin{array}{c}
n-m \\
\mathbf{y}_{i}
\end{array} \times{ }^{k-m} \mathbf{y}_{j}\right)+c^{i j}\left(\begin{array}{l}
m \\
\mathbf{y}_{i}
\end{array} \stackrel{k-m}{\mathbf{y}}_{j}\right)\right) .
$$

В силу рівностей (1.1) попередні вирази $\delta^{k} \mathbf{n}$ запишуться так:

$$
\delta^{k} \mathbf{n}=\left(\begin{array}{c}
k \\
c_{\beta \alpha} \hat{T}^{\alpha \beta}+\frac{1}{2} \delta^{k} c^{i j} c_{i j}
\end{array}\right) \mathbf{n}+c_{s \alpha} \mathbf{r}^{\mathbf{s}} \stackrel{k}{\alpha}^{\alpha}+\stackrel{k}{\mathbf{s}}, \quad k=\overline{1, n}
$$

Домноживши обидві частини цих рівностей на $c^{\beta t} \mathbf{r}_{\beta}$, знайдемо представлення функцій $\stackrel{k}{T^{\alpha}}$ через варіації орта нормалі $S$ :

$$
\stackrel{k}{T^{\alpha}}=c^{\alpha \beta} \mathbf{r}_{\beta} \delta^{k} \mathbf{n}+c^{\beta \alpha} \underset{\mathbf{r}_{\beta} \mathbf{s}, \quad k=\overline{1, n}}{\stackrel{k}{1, n}}
$$

Отже, отримані попередні результати можна сформулювати так:

Теорема 4.1. Овалоїд класу $D_{l+5, p}, l \geqslant 0, p>2 \ll y$ иілому» допускає з.н.м. дебормацію скінченного порядку $n$ з векторами зміщення $\stackrel{k}{\mathbf{y}}\left(x^{1}, x^{2}\right)$ класу $C_{\lambda}^{l+3}(E), \lambda=\frac{p-2}{p}$, яка однозначно визначається заздалегідъ заданими варіачіями елемента площі $\delta^{k} d \sigma \in D_{l+3, p}(E)$ та варіаціями орта нормалі $\delta^{k} \mathbf{n} \in D_{l+5, p}(E)$, причому овалоїд буде жсорстким 
відносно з.н.м. деформацій тоді $і$ тілъки тоді, коли варіацї орта нормалі і елемента площі тотожно дорівнюють нулю.

Слід відзначити, що ця теорема узагальнює результати, отримані в роботах $[15,8,10,5]$.

\section{5. ІСНУВАННЯ З.Н.М. ДЕФОРМАЦІЙ СФЕРИ}

В якості прикладу розглянемо сферу радіуса $R$ :

$$
\mathbf{r}=\left(\frac{2 R x}{1+x^{2}+y^{2}}, \frac{2 R y}{1+x^{2}+y^{2}}, \frac{R\left(1-x^{2}-y^{2}\right)}{1+x^{2}+y^{2}}\right)
$$

де $x=x^{1}, y=x^{2}$. Задамо на сфері $k$-ті варіації орта нормалі і елемента площі поверхні наступним чином:

$$
\begin{aligned}
\delta^{k} \mathbf{n} & =\frac{1}{k^{2}}\left(\frac{x\left(1-x^{2}-y^{2}\right)^{2}}{R\left(1+x^{2}+y^{2}\right)^{3}}, \frac{y\left(1-x^{2}-y^{2}\right)^{2}}{R\left(1+x^{2}+y^{2}\right)^{3}}, \frac{-4\left(x^{2}+y^{2}\right)\left(1-x^{2}-y^{2}\right)}{R\left(1+x^{2}+y^{2}\right)^{3}}\right)+\stackrel{k}{\mathbf{s}}, \\
\delta^{k} d \sigma & =\left(\stackrel{k}{N}-\frac{1}{k^{2}} \frac{8 R^{2}}{\left(1+x^{2}+y^{2}\right)^{2}}\right), \quad k=\overline{1, n}
\end{aligned}
$$

де $\stackrel{k}{N}$ мають вигляд (4.1).

Згідно з рівностями $(4.2)$ i $(4.3)$, функції $T^{\alpha}$ i $\stackrel{k}{\mu}$ на сфері матимуть вигляд:

$$
\stackrel{k}{T^{1}}=\frac{y\left(1-x^{2}-y^{2}\right)}{2 R^{2}\left(1+x^{2}+y^{2}\right)} \quad \frac{1}{k^{2}}, \quad \stackrel{k}{2}=-\frac{x\left(1-x^{2}-y^{2}\right)}{2 R^{2}\left(1+x^{2}+y^{2}\right)} \quad \frac{1}{k^{2}}, \quad \stackrel{k}{\mu}=\frac{1}{k^{2}},
$$

де $k=\overline{1, n}$. Тоді $\stackrel{k}{T^{\alpha}}{ }_{, \alpha}=0$, а система рівнянь $(2.3)$ в даному випадку матиме наступне представлення:

$$
\frac{\partial \stackrel{k}{\omega}}{\partial \bar{z}}=-\frac{4 i \sqrt{R} \bar{z}(1-z \bar{z})}{(1+z \bar{z})^{5}} \frac{1}{k^{2}}, \quad k=\overline{1, n} .
$$

Ïї загальний розв'язок можна записати так:

$$
\stackrel{k}{\omega}=-\frac{1}{\pi k^{2}} \iint_{E} \frac{4 i \bar{\zeta} \sqrt{R}(1-\zeta \bar{\zeta})}{(1+\zeta \bar{\zeta})^{5}(\zeta-z)} d \zeta d \eta, \quad \zeta=\eta+i \varsigma
$$

оскільки в даному випадку $B=0$.

Скориставшись диференціальною властивістю оператора $T_{f}$ :

$$
\frac{\partial T_{f}}{\partial \bar{z}}=\frac{\partial}{\partial \bar{z}}\left(-\frac{1}{\pi} \iint_{E} \frac{f}{\zeta-z} d \zeta d \eta\right)=f
$$


знайдемо функції $\stackrel{k}{\omega}$ :

$$
\stackrel{k}{\omega}=\frac{2 i \sqrt{R} \bar{z}^{2}}{(1+z \bar{z})^{4}} \frac{1}{k^{2}}, \quad k=\overline{1, n} .
$$

В силу рівностей $(2.2),(2.1),(1.2)$ із (1.4) отримаємо явний вираз векторів зміщення, які симетрично залежать від змінних $x$ та $y$ :

$$
\stackrel{k}{\mathbf{y}}=\frac{1}{k^{2}} \mathbf{a}+\stackrel{k}{\mathbf{c}_{0}}, \quad k=\overline{1, n}
$$

де координати $\mathbf{a}\left(a_{1}, a_{2}, a_{3}\right)$ мають представлення:

$$
\begin{gathered}
a_{1}=\frac{x\left(x^{2}+y^{2}\right)-2 R x\left(1+x^{2}+y^{2}\right)^{2}}{\left(1+x^{2}+y^{2}\right)^{3}}, \\
a_{2}=\frac{y\left(x^{2}+y^{2}\right)-2 R y\left(1+x^{2}+y^{2}\right)^{2}}{\left(1+x^{2}+y^{2}\right)^{3}}, \\
\left.a_{3}=\frac{1+x^{2}+y^{2}+2\left(x^{2}+y^{2}\right)^{2}-2 R\left(1-x^{2}-y^{2}\right)\left(1+x^{2}+y^{2}\right)^{2}}{\left(1+x^{2}+y^{2}\right)^{3}}\right),
\end{gathered}
$$

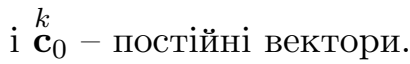

Отже, сфера «у цілому» допускає нетривіальну н.м. деформацію скінченного порядку $n$ з векторами зміщення вигляду (5.3), яка визначається заздалегідь заданими варіаціями елемента площі та орта нормалі, які мають представлення (5.1), (5.2) відповідно.

Підсумовуючи викладене вище, робимо наступний висновок. Регулярний овалоїд «у иілому» допускає з.н.м. деформацію скінченного порядку $n$, яка визначаєтъся заздалегідъ заданими 3 ф функиіями. Пояснено їх геометричний сенс. Отримані результати проілюстровані на сбері. Розроблені методи дозволяють застосовувати їх при вивченні дебормації інших поверхонь, які нерідко використовуються в різних сферах повсякденного життя людства.

\section{ЛIтерАТУРA}

[1] I. Hinterleitner, V. Kiosak. $\varphi$ (ric)-vector fields on conformally flat spaces. Proceedings of American Institute of Physics, 1191:98-103, 2009, doi: 10.1063/1.3275604.

[2] V. Kiosak, V. Matveev. There exist no 4-dimensional geodesically equivalent metrics with the same stress-energy tensor. J. Geom. Phys., 78:1-11, 2014, doi: $10.1016 / j$.geomphys.2014.01.002.

[3] Yu. Vashpanov, Jung-Young Son, Gwanghee Heo, Tatyana Podousova, Yong Suk Kim. Determination of geometric parameters of cracks in concrete by image processing. Advances in Civil Engineering, 2019:1-14, October 2019, doi: 10.1155/2019/2398124.

[4] N. Vashpanova, T. Podousova, Ju. Fedchenko. Canonical deformations of pseudo-riemannian spaces. AIP Conference Proceedings 2164, 040005, 2019, doi: $10.1063 / 1.5130797$. 
[5] Л. Безкоровайна. Структура множини розв'язків системи рівнянь для загальної нескінченно малої деформації. Тези доповідей міжнародної конференцї̈ "Геометрія в Одесі-2004 Одеса, pages 7-8, 2004.

[6] Л. Безкоровайна. Поверхности, образованные действительной и мнимой частями аналитической функции: А-деформации, происходящие независимо или одновременно. Українсъкий математичний журнал, 70(4):447-463, 2018.

[7] Л. Безкоровайна, Т. Вашпанова. $A$-деформації поверхні зі стаціонарною довжиною $L G T$-ліній. Українсъкий математичний журнал, 62(7):878-884, Лип 2010, doi: $10.1007 / \mathrm{s} 11253-010-0410-\mathrm{y}$.

[8] Л. Безкоровайная. О бесконечно малых ареальных деформациях овальных поверхностей. Известия вузов, Математика, 5(252):69-71, 1983.

[9] Илья Векуа. Обобщенные аналитические функиии. М.: Наука. 509 с., 1988.

[10] Нина Дерманец. Определение А-деформаций высших порядков по заданным значениям вариаций нормали. УкрНИИНТИ.-№56, Ук-84 Деn.-40 c.- Om.13.01.84, 1984.

[11] Николай Ефимов. Качественные вопросы теории деформачий поверхностей, volume 2 of Pure and applied mathematics. УМH. T.III, 1948.

[12] Дмитрий Жуков. Бесконечно малые MG-деформации овалоидов. Владикавказский матем. журнал, 15(2):36-45, 2013.

[13] Борис Караев. Бесконечно малые изгибания высших порлдков в тензорном изложении., volume 6. Известия АН Туркменской СCР, 1969.

[14] Сергей Климентов. О продолжсении бесконечно малых изгибаний высших порядков односвязной поверхности положительной кривизны., volume 36. Мат. заметки, 1984.

[15] Павел Колобов. О бесконечно малых деформациях поверхности с сохранением площади. Ученые записки Кабардино-Балкарского ун-та, сер. физика, матем., 30:65-58, 1966.

[16] Павел Марков. Бесконечно малые изгибания высших порядков многомерных поверхностей., volume 8. Укр. геом. сб., 1982.

[17] Т. Подоусова, Н. Вашпанова. О продолжении А-деформаций поверхностей положительной кривизны с краем. Proceedings of the International Geometry Center, 7(3):38-48, 2014, doi: 10.15673/2072-9812.3/2014.40572.

[18] Валентин Фоменко. О жесткости овалоидов относительно g-деформаций при условии стационарности заданной функции главных радиусов кривизны. Современные проблемы математики и механики. - М.: Из-во МГУ, VI Математика:(3):177-186, 2011.

Надійшла до редакцї 19 грудня 2019, прийнята до друку 26 лютого 2020.

Т. Ю. Подоусова

ОДЕСЬКА ОБЛАСТЬ, ОВІДІОПОЛЬСЬКИЙ РАЙОН, С. ЛИМАНКА, ЖМ. УЛЬЯНІВКА, МАСИВ

РАДУжний, БУд.6, кв.36

Email: tatyana_top@ukr.net

ORCID: orcid.org/0000-0002-9492-126X

Н. В. Вашпанова

М. ОДЕССА, ВУЛ. АКАДЕМІКА КОРОЛЕВА 79/1, КВ.10

Email: vasha_nina@ukr.net 